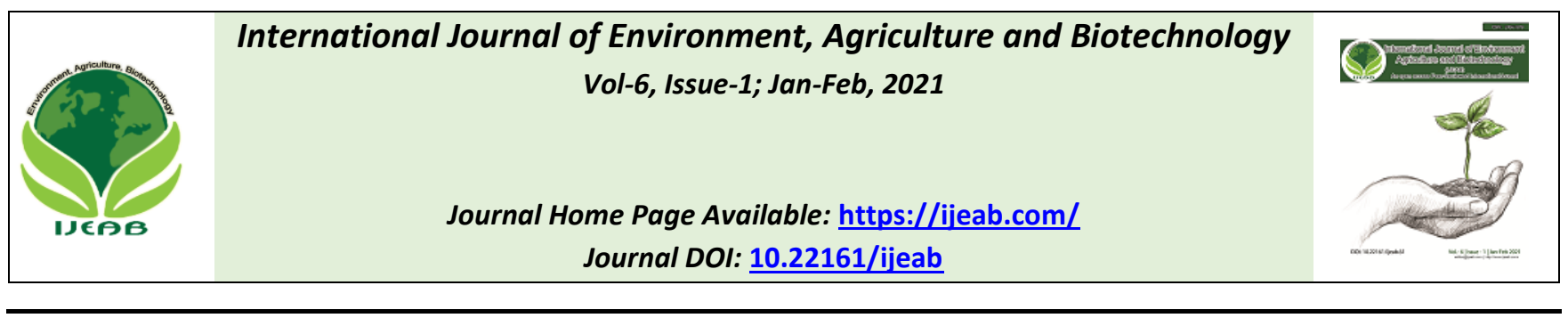

\title{
Identification of The Potential of Degrading Carrageenan in Red Algae Kappaphycus alvarezii Symbiotic Bacteria
}

\author{
Andi Alya Yusriyyah*, Asmi Citra Malina AR Tassakka, Gunarto Latama
}

Department of Marine Science and Fisheries, Hasanuddin University, Indonesia

*Corresponding author

Received: 28 Oct 2020; Received in revised form: 06 Jan 2021; Accepted: 16 Jan 2021; Available online: 29 Jan 2021

(C)2021 The Author(s). Published by Infogain Publication. This is an open access article under the CC BY license

(https://creativecommons.org/licenses/by/4.0/).

\begin{abstract}
Kappahycus alvarezii is a red alga contained large amount of bioactive material, such as carrageenan. Carrageenan is useful as a raw material for several industries and can be degraded by marine bacteria through breaking the linkages in polysaccharide carrageenan into oligosaccharide carrageenan. The aim of this study is identification of degrading carrageenan in K. alvarezii symbiotic bacteria. The results showed there was 14 isolate bacteria, and all of the isolates have clear zone on congo red staining activity. The isolate bacteria were 7 genera as K. alvarezii symbiotic bacteria, such as Labrenzia sp., Alteromonas sp., Vibrio sp., Celeribacter sp., Pseudoalteromonas sp., Phaeobacter sp. and Cobetia sp. Labrenzia sp., Alteromonas sp., Vibrio sp., Pseudoalteromonas sp., Phaeobacter sp. were recognized to have strong interactions with carrageenan in red algae, while the other Celeribacter sp. and Cobetia sp. have strong interactions with alginate in brown algae.
\end{abstract}

Keywords - Kappahycus alvarezii, carrageenan, degrading bacteria.

\section{INTRODUCTION}

Kappahycus alvarezii is red algae that generally has cylindric thallus, smooth surface, cartilaginous and consists of several types based on the color, such as green, yellowish green, gray, brown and red (Parenrengi et al., 2010). K. alvarezii was lived in tidal habitats, coral reef flats and attached to hard substrates (Erlania, 2013). K. alvarezii was contained large amount of carrageenan and used as stabilizer and gelling agent in processed meat, ice cream, chocolate, pudding, pet food, shampoo, toothpaste and cleaning products industrial (Hotchkiss et al., 2016; Barret, 2018).

In the ecosystem, bacteria were played an important role because of its ability to degrade organic matters to inorganic matters (Ginting et al., 2019). The existence of symbiont bacteria was to protect their host and produce secondary metabolites (Funty, 2015). The use of secondary metabolites in algae, for example as bioactive materials (Nurhaedar, 2008). Carrageenan as a bioactive material in algae can be degraded by marine bacteria, especially gram- negative bacteria and produced enzymes to degrade carrageenans and it was useful for several industries (Chauhan \& Saxena, 2016). Carrageenans degradation was a process to break the linkages in polysaccharide carrageenans to be oligosaccharide carrageenan with low molecular weight (Ghanbarzadeh et al., 2018). Several studies showed that Pseudoalteromonas sp. (Li et al., 2013); Tamlana sp. was isolated from red algae Hyalosiphonia caespitosa (Sun et al., 2010) and Cythopaga sp. was isolated from red algae Eucheuma gelutinue (Mou et al., 2004) have degrading carrageenan ability. Based on previous several studies, there is no $K$. alvarezii symbiotic bacteria in degrading carrageenan. Therefore, it is necessary to conduct research on the identification of degrading carrageenans in $K$. alvarezii symbiotic bacteria.

\section{MATERIAL AND METHODS}

Kapphycus alvarezii AND CARRAGEENAN USED IN THIS STUDY 
Algae $K$. alvarezii were collected from USA Marine Biological Institute, Kochi University, Japan and floated in Uranouchi Bay, Tosa, Kochi Prefecture, Japan for one week to collect the bacteria. While several commercial carrageenan was used in this study, $\kappa$-carrageenan and $\lambda$ carrageenan purchased from Wako.

\section{ISOLATION OF $K$. alvarezii SYMBIOTIC- BACTERIA}

Arificial water agar medium was made with adding $0.5 \%$ carrageenan and incubated with red algae $K$. alvarezii for 3 days. After 3 days, the artificial seawater bacteria were dropped around $0.1 \mathrm{ml}$ in marine broth agar. Moreover, bacteria were incubated at $25^{\circ} \mathrm{C}$ for 2 days to get the pure bacteria cultured.

\section{DEGRADING CARRAGEENAN SCREENING BY CONGO RED STAINING}

Bacteria in marine broth agar medium was inoculated to marine broth medium and incubated at $25^{\circ} \mathrm{C}$ for 2 days. Congo red agar medium was made from 4 gr gar medium, $25 \mathrm{ml} 0.5 \%$ carrageenan and $1 \mathrm{ml}$ congo red. Bacteria colonies from marine broth medium was dropped into congo red agar medium for $0.1 \mathrm{ml}$. The bacteria were incubated at $25^{\circ} \mathrm{C}$ for 2 days to identify the clear zone. Formed clear zone was an indicator of the carrageenan degrading activity existence. Bacteria with clear zone was inoculated and analyzed by $16 \mathrm{~S}$ rDNA.

\section{S rDNA ANALYSIS OF $K$. alvarezii SYMBIOTIC- BACTERIA}

A colony of 14 isolate bacteria were used as a template for PCR. Isolate bacteria were amplified by using universal primer proR2 (5'AGAGTTTGATCMTGGCTCAG-3') dan 534R (5'ATTACCGCGGCTGCTGG-3'). PCR products were applied to agarose gel electrophoresis and purified using Wizard® SV Gel and PCR Clean-Up System (Promega). The purified DNA were sequenced by ABI PRISM® 3130 Genetic Analyzer (Applied Biosystems Japan) using BigDye ${ }^{\circledR}$ Terminator v3.1 and analyzed with BLAST on NCBI.

\section{RESULT AND DISCUSSION}

Bacteria isolation found 14 isolate bacteria with big size colonies. Congo red staining activity showed that there was clear zone in 14 isolate bacteria (Fig 1). Congo red was used for degrading carrageenans activity because congo red have strong interaction with polysaccharide which contained cellulose linked by $\beta$-1,4-glycosidic linkages (Teather \& Wood, 1982). FAO (2003) was explained that red algae contain carrageenan and cellulose which insoluble in water and alkali. The clear zone was formed because of the reaction between congo red and $\beta$ 1,4-glycosidic linkages in cellulose polymer (Missa et al., 2016).
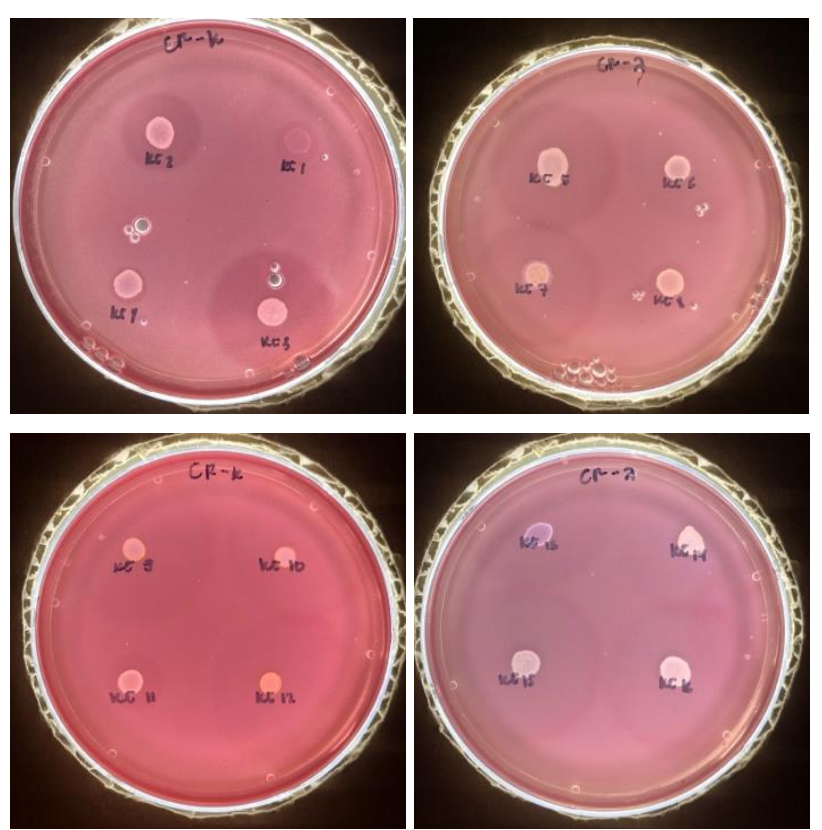

Fig 1: Congo red staining activity on 14 isolate bacteria

Electrophoresis showed that the measurement of bacteria DNA fragment was about $500 \mathrm{bp}$ and it was compared to the marker $1 \mathrm{kbp}$ DNA (Fig 2). The purification of DNA was measured by absorbance $260 \mathrm{~nm}$ and $280 \mathrm{~nm}$. It showed that the absorbance of purified DNA was about 1.73-2.07 (Table 1). Thermo Fisher Scientific (2010) explained that the ratio of absorbance $260 \mathrm{~nm}$ and $280 \mathrm{~nm}$ was about 1.8 and it was "pure" for DNA purification.

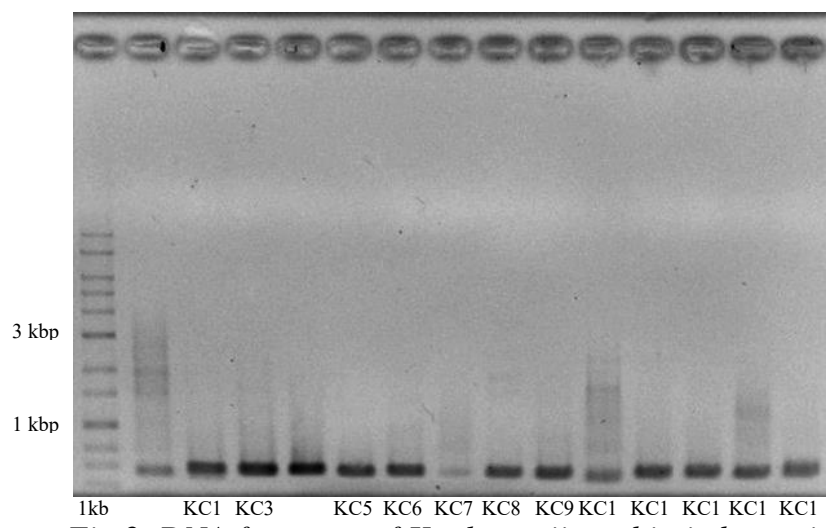

Fig 2. DNA fragment of $K$. alvarezii symbiotic bacteria

Table 1. Absorbance of K. alvarezii symbiotic bacteria DNA purification

\begin{tabular}{|l|l|}
\hline Bacteria & $\mathbf{A}_{260 / 280}$ \\
\hline
\end{tabular}




\begin{tabular}{|c|c|}
\hline Isolate 1 & 1.91 \\
\hline Isolate 2 & 1.95 \\
\hline Isolate 3 & 1.91 \\
\hline Isolate 4 & 2 \\
\hline Isolate 5 & 2.04 \\
\hline Isolate 6 & 1.9 \\
\hline Isolate 7 & 1.73 \\
\hline Isolate 8 & 1.93 \\
\hline Isolate 9 & 1.89 \\
\hline Isolate 10 & 1.76 \\
\hline Isolate 11 & 2.07 \\
\hline Isolate 12 & 1.97 \\
\hline Isolate 13 & 1.93 \\
\hline Isolate 14 & 1.82 \\
\hline
\end{tabular}

\begin{tabular}{|c|c|c|c|}
\hline & & & MT307282 \\
\hline $\begin{array}{c}\text { Isolate } \\
5\end{array}$ & Alteromonas sp. & $100 \%$ & $\begin{array}{c}\text { Alteromonas } \\
\text { macleodii } \\
\text { ROA033, } \\
\text { Accession No. } \\
\text { MT515801 }\end{array}$ \\
\hline $\begin{array}{c}\text { Isolate } \\
6\end{array}$ & Vibrio sp. & $99.80 \%$ & $\begin{array}{c}\text { Vibrio rotiferanus } \\
\text { AM7, Accession } \\
\text { No. AP019798 }\end{array}$ \\
\hline $\begin{array}{c}\text { Isolate } \\
7\end{array}$ & Celeribacter sp. & $100 \%$ & $\begin{array}{c}\text { Celereibacter } \\
\text { naphthalenivorans } \\
\text { EMB201, } \\
\text { Accession No. } \\
\text { NR_137260 }\end{array}$ \\
\hline $\begin{array}{l}\text { Isolate } \\
8\end{array}$ & $\begin{array}{l}\text { Pseudoalteromonas } \\
\text { sp. }\end{array}$ & $100 \%$ & $\begin{array}{c}\text { Pseudoalteromonas } \\
\text { sp. L10, Accession } \\
\text { No. MN889153 }\end{array}$ \\
\hline $\begin{array}{c}\text { Isolate } \\
9\end{array}$ & $\begin{array}{l}\text { Pseudoalteromonas } \\
\text { sp. }\end{array}$ & $100 \%$ & $\begin{array}{c}\text { Pseudoalteromonas } \\
\text { sp. S4498, } \\
\text { Accession No. } \\
\text { MT514367 }\end{array}$ \\
\hline $\begin{array}{c}\text { Isolate } \\
10\end{array}$ & Phaeobacter sp. & $99.33 \%$ & $\begin{array}{c}\text { Uncultured } \\
\text { bacterium 5M23, } \\
\text { Accession No. } \\
\text { JF272132 }\end{array}$ \\
\hline $\begin{array}{c}\text { Isolate } \\
11\end{array}$ & $\begin{array}{l}\text { Pseudoalteromonas } \\
\text { sp. }\end{array}$ & $100 \%$ & $\begin{array}{c}\text { Pseudoalteromonas } \\
\text { sp. Md236, } \\
\text { Accession No. } \\
\text { AY461673 }\end{array}$ \\
\hline $\begin{array}{c}\text { Isolate } \\
12\end{array}$ & Vibrio sp. & $100 \%$ & $\begin{array}{c}\text { Vibrio campbellii } \\
1511126, \\
\text { Accession No. } \\
\text { CP025953 }\end{array}$ \\
\hline $\begin{array}{c}\text { Isolate } \\
13\end{array}$ & Cobetia sp. & $100 \%$ & $\begin{array}{c}\text { Cobetia pacifica } \\
\text { GPM2, Accession } \\
\text { No. CP047970 }\end{array}$ \\
\hline $\begin{array}{c}\text { Isolate } \\
14\end{array}$ & Alteromonas sp. & $100 \%$ & $\begin{array}{l}\text { Alteromonas } \\
\text { macleodii } \\
\text { ROA033, } \\
\text { Accession No. } \\
\text { MT515801 }\end{array}$ \\
\hline
\end{tabular}

$16 \mathrm{~S}$ rDNA showed that there were 7 genera of symbiotic bacteria in $K$. alvarezii, such as Labrenzia sp., Alteromonas sp., Vibrio sp., Celeribacter sp., Pseudoalteromonas sp., Phaeobacter sp. and Cobetia sp. (Table 2). Azizi et al., (2018) found some bacteria was associated with 4 types of $K$. alvarezii and classified by 11 genera, such as Alteromonas sp., Aestuariibacter sp., Idiomarina sp., Jejuia sp., Halomonas sp., Primoskyibacter sp., Pseudoalteromonas sp., Ruegeria sp., Terasakiella sp., Thalassospira sp. and Vibrio sp. All of bacteria in this study was gram-negative bacteria and have carrageenan degrading ability by congo red staining activity. Chauhan \& Saxena (2016) explained that carrageenase enzyme was only produced extracellularly by gram-negative bacteria.

Table 2. 16S rDNA of K. alvarezii symbiotic bacteria

\begin{tabular}{|c|c|c|c|}
\hline \multicolumn{2}{|c|}{$\begin{array}{c}\text { K. alvarezii symbiotic } \\
\text { bacteria }\end{array}$} & Identity & Reference \\
\hline $\begin{array}{l}\text { Isolate } \\
1\end{array}$ & Labrenzia sp. & $99.77 \%$ & $\begin{array}{l}\text { Labrenzia sp. } \\
\text { THAF35, } \\
\text { Accession No. } \\
\text { CP045380 }\end{array}$ \\
\hline $\begin{array}{c}\text { Isolate } \\
2\end{array}$ & Alteromonas sp. & $98.75 \%$ & $\begin{array}{c}\text { Alteromonas tagae, } \\
\text { Accession No. } \\
\text { NR_043977 }\end{array}$ \\
\hline $\begin{array}{l}\text { Isolate } \\
3\end{array}$ & Alteromonas sp. & $99.58 \%$ & $\begin{array}{c}\text { Alteromonas tagae, } \\
\text { Accession No. } \\
\text { NR_043977 }\end{array}$ \\
\hline $\begin{array}{c}\text { Isolate } \\
4\end{array}$ & Vibrio sp. & $99.60 \%$ & $\begin{array}{c}\text { Vibrio campbellii } \\
\text { MMRF1060, } \\
\text { Accession No. }\end{array}$ \\
\hline
\end{tabular}

The first Labrenzia sp. in red algae was Labrenzia polysiphoniae in red algae Polysiphonia sp. (Romanenko et al., 2019). Alteromonas sp. was found as Kappahycus alvarezii symbiotic bacteria and showed a pathogenetic. Alteromonas sp. was able to be pathogen agent that caused ice-ice symptoms (Syafitri et al., 2017). On the other hand, 
Alteromonas sp. showed a potential to degrade some polysaccharide, such as alginate (Neumann et al., 2015), ulvan (Koch et al., 2019); agar (Wang et al., 2005); 1karagenan (Barbeyron et al., 2019) and $\kappa$-karagenan (Barbeyron et al., 1994). Araki et al., (1999) and Zhu \& Ning (2016) found high activity of $\kappa$-carrageenase enzyme through Vibrio sp. purification. Moreover, Pseudoalteromonas sp. had an ability to utilize $\kappa$ carrageenan and $\mathrm{l}$-carrageenan for their energy source (Hettle et al., 2019). Pseudoalteromonas sp. was also degraded $\kappa$-carrageenan (Liu et al., 2011) and $\lambda$ carrageenan (Guibet et al., 2007). Furthermore, Phaeobacter inhibens was found in red algae Tichocarpus crinitus to degrade the carrageenan (Kalitnik et al., 2017). Based on the several studies, Labrenzia sp., Alteromonas sp., Vibrio sp., Pseudoalteromonas sp. and Phaeobacter sp. were recognized to have strong interactions with red algae through the utilization of red algae carrageenan. Whereas Celeribacter sp. and Cobetia sp. was found in brown algae through the utilization of brown algae alginate. Ihua et al. (2020) showed that Celeribacter sp. was found on brown algae thallus Laminaria digitata and Yagi et al. (2016) explained that Cobetia sp. was isolated from brown algae Padina arborescens with alginate degrading enzyme.

\section{CONCLUSION}

In this study, we found 7 genera of Kappahycus alvarezii symbiotic bacteria, such as Labrenzia sp., Alteromonas sp., Vibrio sp., Celeribacter sp., Pseudoalteromonas sp., Phaeobacter sp. and Cobetia sp. All of the bacteria showed an activity on congo red staining based on the formed clear zone. The clear zone was indicated the carrageenan degrading activity.

\section{REFERENCES}

[1] Parenrengi, A., Rachmansyah dan E. Suryati. 2010. Budi Daya Rumput Laut Penghasil Karaginan (Karginofit). Badan Penelitian dan Pengembangan Kelautan dan Perikanan. Jakarta: Kementerian Kelautan dan Perikanan Indonesia

[2] Erlania. 2013. Potensi Budidaya Rumput Laut Kappaphycus alvarezii dan Gracilaria gigas dalam Penyerapan Karbon. Thesis. Institut Pertanian Bogor

[3] Hotchkiss, S., M. Brooks, R. Campbell, K. Philp, A. Tirius. 2016. The use of carrageenan in food. Carrageenans: Sources and Extraction Methods, Molecular Structure, Bioactive Properties and Health Effects. Pp 229-243

[4] Barrett, B. (2018). Viral Upper Respiratory Infection. Integrative Medicine, 170-179.e7. doi:10.1016/b978-0-32335868-2.00018-9

[5] Ginting, E.L., L. Rangian, L.L. Wantania, S. Wullur. 2019. Isolation of symbiotic bacteria with red algae from
Tongkaina Waters, North Sulawesi. Jurnal Ilmiah Platax. 7(2): 394-400

[6] Funty. 2015. Potensi Antibakteri dari Ekstrak Kasar Bakteri Asosiasi Karang Batu yang Terinfeksi Penyakit Brown Band (BrB) Terhadap Bakteri Patogen Staphylococcus aureus dan Escherichia coli. Skripsi- Universitas Hasanudin. Makasar. 39 hal.

[7] Nurhaedar. 2008. Potensi Bakteri Simbion Spons Class Demospongiae sebagai Sumber Senyawa Antimikroba. Makalah Poster Seminar Nasional Persatuan Biologi Indonesia Ke- XIX, Universitas Hasanuddin Makassar

[8] Chauhan, P. S., \& Saxena, A. 2016. Bacterial carrageenases: an overview of production and biotechnological applications. 3 Biotech, 6(2), 1-18. https://doi.org/10.1007/s13205-016-0461-3

[9] Ghanbarzadeh, M., Golmoradizadeh, A., \& Homaei, A. 2018. Carrageenans and carrageenases: versatile polysaccharides and promising marine enzymes. Phytochemistry Reviews, 17(3), 535-571. https://doi.org/10.1007/s11101-018-9548-2

[10] Li, S., Jia, P., Wang, L., Yu, W., \& Han, F. 2013. Purification and characterization of a new thermostable $\kappa$-carrageenase from the marine bacterium Pseudoalteromonas sp. QY203. Journal of Ocean University of China, 12(1), 155-159. doi:10.1007/s11802-013-1994-2

[11] Sun, F., Ma, Y., Wang, Y., \& Liu, Q. (2010). Purification and characterization of novel $\kappa$-carrageenase from marine Tamlana sp. HC4. Chinese Journal of Oceanology and Limnology, 28(6), 1139-1145. doi:10.1007/s00343-0109012-7

[12] Mou, H., Jiang, X., Liu, Z., \& Guan, H. (2004). Structural analysis of kappa-carrageenan oligosaccharides released by carrageenase from marine Cytophaga MCA-2. Journal of Food Biochemistry, 28(3), 245-260. doi:10.1111/j.17454514.2004.tb00068.x

[13] Teather, R.M., P.J. Wood. 1982. Use of congo redpolysaccharide interactions in enumeration and characterization of cellulolytic bacteria from the Bovine Rumen. Applied and Environmental Microbiology. 43(4): 777-780

[14] Missa, H., A. Susilowati, R. Setyaningsih. 2016. Diversity and phylogenetic relationship of cellulolytic bacteria from the feces of Bali Cattle in South Central Timor, East Nusa Tenggara, Indonesia. Biodiversitas. 17(2): 614-619

[15] Thermo Fisher Scientific. 2010. NanoDrop 1000 Spectrophotometer V3.8 User's Manual.

[16] Azizi, A., Mohd Hanafi, N., Basiran, M. N., \& Teo, C. H. 2018. Evaluation of disease resistance and tolerance to elevated temperature stress of the selected tissuecultured Kappaphycus alvarezii Doty 1985 under optimized laboratory conditions. 3 Biotech, 8(8), 321. https://doi.org/10.1007/s13205-018-1354-4

[17] Romanenko, L. A., Kurilenko, V. V., Guzev, K. V., \& Svetashev, V. I. 2019. Characterization of Labrenzia polysiphoniae sp. nov. isolated from red alga Polysiphonia sp. Archives of Microbiology. doi:10.1007/s00203-01901640-0 
[18] Syafitri, E., Prayitno, S. B., Ma'ruf, W. F., \& Radjasa, O. K. 2017. Genetic diversity of the causative agent of ice-ice disease of the seaweed Kappaphycus alvarezii from Karimunjawa island, Indonesia. IOP Conference Series: Earth and Environmental Science, 55, 012044

[19] Neumann, A. M., Balmonte, J. P., Berger, M., Giebel, H.A., Arnosti, C., Voget, S., Wietz, M. 2015. Different utilization of alginate and other algal polysaccharides by marineAlteromonas macleodiiecotypes. Environmental Microbiology, 17(10), 3857-3868. doi:10.1111/14622920.12862

[20] Koch, H., Freese, H. M., Hahnke, R. L., Simon, M., \& Wietz, M. 2019. Adaptations of Alteromonas sp. 76-1 to Polysaccharide Degradation: A CAZyme Plasmid for Ulvan Degradation and Two Alginolytic Systems. Frontiers in Microbiology, 10. doi:10.3389/fmicb.2019.00504

[21] Wang, L., Li, S., Zhang, S., Li, J., Yu, W., \& Gong, Q. 2015. A new $\kappa$-carrageenase $\mathrm{CgkS}$ from marine bacterium Shewanella sp. Kz7. Journal of Ocean University of China, 14(4), 759-763. doi:10.1007/s11802-015-2713-y

[22] Barbeyron T, Zonta E, Le Panse S, Duchaud E, Michel G. 2019. Alteromonas fortis sp. nov., a non-flagellated bacterium specialized in the degradation of iotacarrageenan, and emended description of the genus Alteromonas. Int J Syst Evol Microbiol. 2019 Aug;69(8):2514-2521. doi: 10.1099/ijsem.0.003533. Epub 2019 Jun 14. PMID: 31199221.

[23] Barbeyron, T., Henrissat, B., \& Kloareg, B. 1994. The gene encoding the kappa-carrageenase of Alteromonas carrageenovora is related to $\beta-1,3-1,4$-glucanases. Gene, 139(1), 105-109. doi:10.1016/0378-1119(94)90531-2

[24] Araki, T., Higashimoto, Y., \& Morishita, T. 1999. Purification and Characterization of $\kappa$-Carrageenase from a Marine Bacterium, Vibrio sp. CA-1004. Fisheries Science, 65(6), 937-942. doi:10.2331/fishsci.65.937

[25] Zhu, B and L, Ning. 2016. Purification and characterization of a new $\kappa$-carrageenase from the marine bacterium Vibrio sp. NJ-2. Journal of Microbiology and Biotechnology. 26(2): 255-262

[26] Hettle, A. G., Hobbs, J. K., Pluvinage, B., Vickers, C., Abe, K. T., Salama-Alber, O., McGuire, B. E., Hehemann, J. H., Hui, J., Berrue, F., Banskota, A., Zhang, J., Bottos, E. M., Van Hamme, J., \& Boraston, A. B. 2019. Insights into the $\kappa / \mathrm{l}$-carrageenan metabolism pathway of some marine Pseudoalteromonas species. Communications biology, 2, 474. https://doi.org/10.1038/s42003-019-0721-y

[27] Liu, G.-L., Li, Y., Chi, Z., \& Chi, Z.-M. 2011. Purification and characterization of $\kappa$-carrageenase from the marine bacterium Pseudoalteromonas porphyrae for hydrolysis of א-carrageenan. Process Biochemistry, 46(1), 265 271. doi:10.1016/j.procbio.2010.08.021

[28] Guibet, M., Colin, S., Barbeyron, T., Genicot, S., Kloareg, B., Michel, G., \& Helbert, W. 2007. Degradation of $\lambda$ carrageenan by Pseudoalteromonas carrageenovora $\lambda$ carrageenase: a new family of glycoside hydrolases unrelated to $\kappa$ - and 1 -carrageenases. Biochemical Journal, 404(1), 105-114. https://doi.org/10.1042//bj20061359

[29] Ihua MW, FitzGerald JA, Guihéneuf F, Jackson SA,
Claesson MJ, Stengel DB, Dobson ADW. 2020. Diversity of bacteria populations associated with different thallus regions of the brown alga Laminaria digitata. PLoS One. 15(11):e0242675. doi: 10.1371/journal.pone.0242675. PMID: 33237941; PMCID: PMC7688147.

[30] Yagi, H., Fujise, A., Itabashi, N., \& Ohshiro, T. 2016. Purification and characterization of a novel alginate lyase from the marine bacterium Cobetia sp. NAP1 isolated from brown algae. Bioscience, Biotechnology, and Biochemistry, $\quad$ 80(12), 23382346. doi:10.1080/09168451.2016.1232154 